\title{
On the Principle of Virtual Powers in Continuum Mechanics
}

Received: date / Accepted: date

\begin{abstract}
We give a general formulation of the Principle of virtual powers in Continuum Mechanics from a distributional point of view, and study some of its relevant consequences in the field of balance equations.
\end{abstract}

Keywords Virtual Powers · Contact Interactions · Balance Equations

\section{Introduction}

It is well known that the basic equations of Mechanics can be given starting from the Principle of virtual powers. However, such a procedure is usually deemed to be only instrumental, and the formulation in terms of forces is kept as the reference frame. On the contrary, it is worthwhile considering that the approach based on the concept of virtual power is independent of the notion of force, and it has lots of capabilities and a great appeal. In particular, it enables a more general and versatile treatment of the mechanical problem; this is mainly due to the prerogatives of duality between forces and velocities.

The situation in the specific context of Continuum Mechanics has not been different: the Principle of virtual powers was regarded as a technical tool. Nonetheless, in two important papers of 1973 [4,5] Paul Germain faced the question in a comprehensive way. In particular, the author developed the so called first and second gradient theories, showing the outstanding flexibility of the Principle. We refer also to [7] for a slightly earlier presentation of the topic using the Principle of Virtual Work.

In the present paper, starting from Germain's ideas, we revise the formulation of the Principle of virtual powers. On the base of recent advances in the foundations of Continuum Mechanics such as [1], we can give a more general treatment

Dipartimento di Matematica e Fisica, Università Cattolica del Sacro Cuore, Via dei Musei 41, I-25121 Brescia, Italy

C. Banfi E-mail: c.banfi@dmf.unicatt.it·A. Marzocchi E-mail: a.marzocchi@dmf.unicatt.it·A. Musesti E-mail: a.musesti@dmf.unicatt.it 
of the subject. In particular, the approach by means of the theory of distributions, mentioned by Germain himself but not fully developed, is here adopted from the beginning. Clearly, in order to obtain deeper results such as the Cauchy Stress Theorem, some extra regularity has to be assumed. Note that a power depends in general from two variables, the velocity field and the subbody. So it is a bit more complex than a mere distribution.

Among the results obtained in this paper we point out a careful study of the contact powers, the general treatement of the Principle of virtual powers in the setting of measures (which can be singular with respect to the usual volume and area), and an optimal formulation of the axioms for a first-gradient theory by means of the systematic use of affine functions and $n$-intervals.

\section{Measure-theoretic stuff}

The section acquaints the readers with some detailed results of measure theory which will be useful in the sequel.

We say that a set $M \subseteq \mathbb{R}^{n}$ is normalized, if $M=M_{*}$ where $M_{*}$ denotes the set of points of density of $M$ with respect to the $n$-dimensional Lebesgue measure $\mathscr{L}^{n}$,

$$
M_{*}=\left\{x \in \mathbb{R}^{n}: \mathscr{L}^{n}\left(B_{r}(x) \backslash M\right) / r^{n} \rightarrow 0 \quad \text { as } r \rightarrow 0^{+}\right\} .
$$

The measure-theoretic boundary of a set $M$ is

$$
\partial_{*} M=\mathbb{R}^{n} \backslash\left(M_{*} \cup\left(\mathbb{R}^{n} \backslash M\right)_{*}\right) .
$$

A normalized set is a sort of "open" set from a measure-theoretical viewpoint. However, topological concepts such as "open" or "closed" can be considered in the eyes of Mechanics as external to the model, while measure-theoretic notions are much more related to the notion of "mass", which is with no doubt involved in the model. In the following, the usual topological notions of interior, closure and boundary will be denoted by int, $\mathrm{cl}$ and bd resp.

Hereafter, $\Omega$ will denote a bounded open normalized subset of $\mathbb{R}^{n}$. We denote by $\mathfrak{M}(\Omega)$ the set of Borel measures $\mu: \mathfrak{B}(\Omega) \rightarrow[0,+\infty]$ finite on compact subsets of $\Omega$. Let $\mu \in \mathfrak{M}(\Omega)$ and $\lambda: \mathscr{P} \rightarrow \mathbb{R}$, where $\mathscr{P}$ is a collection of Borel subsets of $\Omega$. We write $\lambda \ll \mu$, if

$$
\forall M \in \mathscr{P}: \mu(M)=0 \quad \Rightarrow \quad \lambda(M)=0 .
$$

In this paper we choose a particularly simple class of subbodies, namely open $n$-intervals with compact closure in $\Omega$. In our opinion, every class of subbodies should contain such a family, which is in a sense minimal. Moreover, since we deal with measures which can be singular, we have to introduce a notion of "almost all" in the class of $n$-intervals. This is the meaning of $G$-interval in Definition 1 below.

Definition 1 A full grid $G$ is an ordered triple

$$
G=\left(x_{0},\left(e_{1}, \ldots, e_{n}\right), \widehat{G}\right),
$$

where $x_{0} \in \mathbb{R}^{n},\left(e_{1}, \ldots, e_{n}\right)$ is a positively oriented orthonormal basis in $\mathbb{R}^{n}$ and $\widehat{G}$ is a Borel subset of $\mathbb{R}$ with $\mathscr{L}^{1}(\mathbb{R} \backslash \widehat{G})=0$. 
A subset $M$ of $\mathbb{R}^{n}$ is said to be $a G$-interval, if

$$
M=\left\{x \in \mathbb{R}^{n}: a_{j}<\left(x-x_{0}\right) \cdot e_{j}<b_{j} \forall j=1, \ldots, n\right\}
$$

for some $a_{1}, b_{1}, \ldots, a_{n}, b_{n} \in \widehat{G}$. We set

$$
\mathscr{M}_{G}=\left\{M \subseteq \mathbb{R}^{n}: M \text { is a } G \text {-interval with } \operatorname{cl} M \subseteq \Omega\right\} .
$$

It is clear that the elements of $\mathscr{M}_{G}$ are normalized; they are also regularly open. However, it can happen that the union of two $G$-intervals $M_{1}, M_{2}$ in not normalized (although it is obviously open), so we will consider the normalized union of such sets, $\left(M_{1} \cup M_{2}\right)_{*}$. It holds that

$$
\left(M_{1} \cup M_{2}\right)_{*}=\operatorname{intcl}\left(M_{1} \cup M_{2}\right),
$$

hence in the case of $G$-intervals the well-known notion of "regularly open set" is recovered by the normalization procedure.

Definition 2 Let $X$ be a linear space. A function $\lambda: \mathscr{M}_{G} \rightarrow X$ is said to be $*-$ additive, if

$$
\lambda\left(\left(M_{1} \cup M_{2}\right)_{*}\right)=\lambda\left(M_{1}\right)+\lambda\left(M_{2}\right)
$$

for every $M_{1}, M_{2} \in \mathscr{M}_{G}$ such that $\left(M_{1} \cup M_{2}\right)_{*} \in \mathscr{M}_{G}$ and $M_{1} \cap M_{2}=\varnothing$.

The following theorems are quite standard in Measure Theory. For a proof of the first, one can refer to [1].

Theorem 1 Let $X$ be a finite-dimensional normed space and $\lambda: \mathscr{M}_{G} \times X \rightarrow \mathbb{R} a$ function such that

1. for every $y \in X, \lambda(\cdot, y)$ is $*$-additive;

2. for every $M \in \mathscr{M}_{G}, \lambda(M, \cdot)$ is linear;

3. there exists $\mu \in \mathfrak{M}(\Omega)$ such that

$$
\forall M \in \mathscr{M}_{G}, \forall y \in X: \quad|\lambda(M, y)| \leqslant|y| \mu(M) .
$$

Then there exists a bounded Borel map A : $\Omega \rightarrow X^{*}$ such that

$$
\forall M \in \mathscr{M}_{G}, \forall y \in X: \quad \lambda(M, y)=\int_{M}\langle A(x), y\rangle d \mu(x) .
$$

Moreover, A is uniquely determined $\mu$-a.e.

The following is an easy generalization of [3, Corollary 2.9.4].

Theorem 2 Let $\lambda: \mathscr{M}_{G} \rightarrow \mathbb{R}$ be $*$-additive and suppose that there exists $\mu \in$ $\mathfrak{M}(\Omega)$ such that $\lambda \ll \mu$.

Then $\lambda \leqslant 0$ if and only iffor $\mu$-a.e. $x_{0} \in \Omega$ one has

$$
\lim _{i \rightarrow \infty} \frac{\lambda\left(M_{i}\right)}{\mu\left(M_{i}\right)} \leqslant 0
$$

whenever $\left(M_{i}\right) \subseteq \mathscr{M}_{G}$ is a sequence of open $n$-cubes with $x_{0} \in M_{i}$ and $\operatorname{diam} M_{i} \rightarrow 0$ as $i \rightarrow \infty$. 
For a proof of the following theorem, see [2, p. 43].

Theorem 3 (Lebesgue-Besicovitch Differentiation Theorem) Let $\mu \in \mathfrak{M}(\Omega)$ and $f \in L_{l o c}^{1}(\Omega ; \mu)$. Then for $\mu$-a.e. $x_{0} \in \Omega$

$$
\lim _{i \rightarrow \infty} \frac{1}{\mu\left(M_{i}\right)} \int_{M_{i}} f d \mu=f\left(x_{0}\right)
$$

whenever $\left(M_{i}\right) \subseteq \mathscr{M}_{G}$ is a sequence of open $n$-cubes with $x_{0} \in M_{i}$ and $\operatorname{diam} M_{i} \rightarrow 0$ as $i \rightarrow \infty$.

Corollary 1 Let $\mu \in \mathfrak{M}(\Omega)$ and $f \in L_{\text {loc }}^{1}(\Omega ; \mu)$ be such that

$$
\forall M \in \mathscr{M}_{G}: \quad \int_{M} f d \mu=0 .
$$

Then $f(x)=0$ for $\mu$-a.e. $x \in \Omega$.

Proof It is an easy consequence of the previous theorem, because the left-hand side of (1) vanishes $\mu$-a.e.

\section{Powers of order one}

We denote with Lin the space of linear functions $A: \mathbb{R}^{n} \rightarrow \mathbb{R}^{n}$. The symmetric and skew-symmetric parts of $A$ will be denoted by $A^{s}$ and $A^{\omega}$, respectively. Let Aff be the collection of all affine functions $\mathbf{v}: \Omega \rightarrow \mathbb{R}^{n}, \mathbf{v}(x)=\mathbf{v}_{0}+\mathrm{F} x$, where $\mathrm{F} \in$ Lin.

Definition 3 We say that a function $P: \mathscr{M}_{G} \times \mathrm{Aff} \rightarrow \mathbb{R}$ is a power of order one, if the following properties hold:

1. for every $\mathbf{v} \in \operatorname{Aff}, P(\cdot, \mathbf{v})$ is $*$-additive;

2. for every $M \in \mathscr{M}_{G}, P(M, \cdot)$ is linear;

3. there exist $\mu_{0}, \mu_{1} \in \mathfrak{M}(\Omega)$ such that

$$
|P(M, \mathbf{v})| \leqslant \int_{M}|\mathbf{v}| d \mu_{0}+\int_{M}|\nabla \mathbf{v}| d \mu_{1}
$$

for every $M \in \mathscr{M}_{G}$ and $\mathbf{v} \in$ Aff.

If in the previous definition one can choose $\mu_{1}=0$, then we will refer to $P$ as $a$ body power.

Remark 1 For a theory of higher order powers, where also edge or vertex interactions are involved, it is natural to weaken estimate (c) (see [1]). Moreover, the class of virtual velocities must contain higher grade polynomials.

A first important result is the following theorem, which gives an integral representation for the power.

Theorem 4 There exist two bounded Borel maps a : $\Omega \rightarrow \mathbb{R}^{n}$ and A : $\Omega \rightarrow$ Lin such that $|\mathbf{a}| \leqslant 1 \mu_{0}$-a.e., $|\mathrm{A}| \leqslant 1 \mu_{1}$-a.e. and

$$
\forall M \in \mathscr{M}_{G}, \forall \mathbf{v} \in \text { Aff : } \quad P(M, \mathbf{v})=\int_{M} \mathbf{a} \cdot \mathbf{v} d \mu_{0}+\int_{M} \mathrm{~A} \cdot \nabla \mathbf{v} d \mu_{1} .
$$

Moreover, a is uniquely determined $\mu_{0}$-a.e. and $\mathrm{A}$ is uniquely determined $\mu_{1}$-a.e. 
Proof One has

$$
\forall M \in \mathscr{M}_{G}, \forall \mathbf{v}_{0} \in \mathbb{R}^{n}:\left|P\left(M, \mathbf{v}_{0}\right)\right| \leqslant \int_{M}\left|\mathbf{v}_{0}\right| d \mu_{0},
$$

hence by Corollary 1 there exists a bounded Borel map a : $\Omega \rightarrow \mathbb{R}^{n}$ such that

$$
\forall M \in \mathscr{M}_{G}, \forall \mathbf{v}_{0} \in \mathbb{R}^{n}: P\left(M, \mathbf{v}_{0}\right)=\int_{M} \mathbf{a} \cdot \mathbf{v}_{0} d \mu_{0}
$$

and $|\mathbf{a}| \leqslant 1 \mu_{0}$-a.e.

Given $\mathrm{F} \in$ Lin and the vector field $\mathbf{v}_{\mathrm{F}}(x)=\mathrm{F} x$, we now want to prove that

$$
\left|P\left(M, \mathbf{v}_{\mathrm{F}}\right)-\int_{M} \mathbf{a} \cdot \mathbf{v}_{\mathrm{F}} d \mu_{0}\right| \leqslant \int_{M}|\mathrm{~F}| d \mu_{1}
$$

for every $M \in \mathscr{M}_{G}$. Consider the function

$$
\lambda_{1}(M)=P\left(M, \mathbf{v}_{\mathrm{F}}\right)-\int_{M} \mathbf{a} \cdot \mathbf{v}_{\mathrm{F}} d \mu_{0}-\int_{M}|\mathrm{~F}| d \mu_{1} .
$$

Then $\lambda_{1}$ is $*$-additive and $\lambda_{1} \ll \mu$, where $\mu=\mu_{0}+\mu_{1}$. For every $x_{0} \in \Omega$ it can be easily checked that

$$
\lambda_{1}(M)=P\left(M, \mathbf{v}_{\mathrm{F}}-\mathrm{F} x_{0}\right)-\int_{M} \mathbf{a} \cdot\left(\mathbf{v}_{\mathrm{F}}-\mathrm{F} x_{0}\right) d \mu_{0}-\int_{M}|\mathrm{~F}| d \mu_{1} .
$$

Let $x_{0} \in \Omega$ and $\left(M_{i}\right)$ a sequence as in Theorem 2; we have

$$
\lim _{i \rightarrow \infty} \frac{\lambda_{1}\left(M_{i}\right)}{\mu\left(M_{i}\right)} \leqslant \lim _{i \rightarrow \infty} \frac{1}{\mu\left(M_{i}\right)} \int_{M_{i}}\left|\mathrm{~F}\left(x-x_{0}\right)\right|(1+|\mathbf{a}|) d \mu_{0}=0
$$

and by Theorem 3 the right-hand side vanishes for $\mu$-a.e. $x_{0} \in \Omega$, hence $\lambda_{1} \leqslant 0$. In the same way, setting

$$
\lambda_{2}(M)=P\left(M, \mathbf{v}_{\mathrm{F}}\right)-\int_{M} \mathbf{a} \cdot \mathbf{v}_{\mathrm{F}} d \mu_{0}+\int_{M}|\mathrm{~F}| d \mu_{1},
$$

one can prove that $\lambda_{2} \geqslant 0$ and (4) follows.

Then, by Corollary 1 there exists a bounded Borel map A : $\Omega \rightarrow$ Lin such that

$$
\forall M \in \mathscr{M}_{G}, \forall \mathrm{F} \in \operatorname{Lin}: P\left(M, \mathbf{v}_{\mathrm{F}}\right)=\int_{M} \mathbf{a} \cdot \mathbf{v}_{\mathrm{F}} d \mu_{0}+\int_{M} \mathrm{~A} \cdot \mathrm{F} d \mu_{1}
$$

and $|\mathrm{A}| \leqslant 1 \mu_{1}$-a.e. Equation (5), combined with (3), ends up the proof.

Now we see that Theorem 4 admits a form of converse.

Proposition 1 Let $\mu_{0}, \mu_{1} \in \mathfrak{M}(\Omega)$ and let $\mathbf{a}: \Omega \rightarrow \mathbb{R}^{n}, \mathrm{~A}: \Omega \rightarrow$ Lin be Borel and bounded.

Then there exists a full grid $G$ such that the function $P: \mathscr{M}_{G} \times \mathrm{Aff} \rightarrow \mathbb{R}$ defined as

$$
P(M, \mathbf{v})=\int_{M} \mathbf{a} \cdot \mathbf{v} d \mu_{0}+\int_{M} \mathrm{~A} \cdot \nabla \mathbf{v} d \mu_{1}
$$

is a power of order one. 
Proof Set $\eta=\mu_{0}+\mu_{1}$ and

$$
\widehat{G}=\left\{a \in \mathbb{R}: \eta\left(\left\{x \in \Omega:\left(x-x_{0}\right) \cdot \mathbf{e}_{i}=a\right\}\right)=0\right\} .
$$

Since $\eta$ is finite on compact subsets of $\Omega$, it is easy to see that the triple $G=$ $\left(x_{0},\left(e_{1}, \ldots, e_{n}\right), \widehat{G}\right)$ is a full grid for any $x_{0} \in \mathbb{R}^{n}$ and $\left(e_{1}, \ldots, e_{n}\right)$. Then it is clear that (6) defines a power.

\subsection{Extension of a power}

We show now that a power, even if it has been defined only on a small class of subbodies and virtual velocities, can indeed be extended in a unique way to (almost all) Borel subsets of $\Omega$ and all vector fields on $\Omega$ of class $C^{1}$. The key tool is the integral representation (2), but one has to pay attention to the $*$-additivity.

We need to introduce the family of almost all Borel sets with compact closure in $\Omega$; for $\eta \in \mathfrak{M}(\Omega)$, we set

$$
\mathscr{B}_{\eta}=\left\{M \subseteq \mathbb{R}^{n}: M=M_{*}, \operatorname{cl} M \subseteq \Omega, \eta\left(\partial_{*} M\right)=0\right\} .
$$

Theorem 5 Let $P$ be a power, $\mathbf{a}, \mathrm{A}$ be as in Theorem 4 and $\eta=\mu_{0}+\mu_{1}$.

Then the function $\tilde{P}: \mathscr{B}_{\eta} \times C^{1}\left(\Omega ; \mathbb{R}^{n}\right) \rightarrow \mathbb{R}$ defined as

$$
\tilde{P}(M, \mathbf{v})=\int_{M} \mathbf{a}(x) \cdot \mathbf{v}(x) d \mu_{0}(x)+\int_{M} \mathrm{~A}(x) \cdot \nabla \mathbf{v}(x) d \mu_{1}(x)
$$

is an extension of $P$ which satisfies $(a),(b)$ and $(c)$ of Definition 3 on all its domain. Moreover, such an extension is unique.

Proof Since $\eta\left(\partial_{*} M\right)=0$ for every $M \in \mathscr{B}_{\eta}$, the function $\tilde{P}$ is $*$-additive on $\mathscr{B}_{\eta}$. Moreover, it clearly satisfies (b) and (c) of Definition 3 on $\mathscr{B}_{\eta} \times C^{1}\left(\Omega ; \mathbb{R}^{n}\right)$.

For the uniqueness, consider a generic function $\tilde{P}: \mathscr{B}_{\eta} \times C^{1}\left(\Omega ; \mathbb{R}^{n}\right) \rightarrow \mathbb{R}$ which satisfies Definition 3 on all its domain with $\eta=\mu_{0}+\mu_{1}$. Then in particular it is a power of order one and there exist two bounded Borel maps $\mathbf{a}, \mathrm{A}$ such that (2) holds. Let $\mathbf{v} \in C^{1}\left(\Omega ; \mathbb{R}^{n}\right)$ and set

$$
\forall M \in \mathscr{B}_{\eta}: \quad \lambda(M)=\tilde{P}(M, \mathbf{v})-\int_{M} \mathbf{a} \cdot \mathbf{v} d \mu_{0}-\int_{M} \mathrm{~A} \cdot \nabla \mathbf{v} d \mu_{1} .
$$

Let $x_{0} \in \Omega,\left(M_{i}\right)$ be a sequence as in Theorem 2 and $\mathbf{w}=\mathbf{v}-\mathbf{v}\left(x_{0}\right)-\nabla \mathbf{v}\left(x_{0}\right)(x-$ $\left.x_{0}\right)$. By (2) it is easy to see that

$$
\lambda(M)=\tilde{P}(M, \mathbf{w})-\int_{M} \mathbf{a} \cdot \mathbf{w} d \mu_{0}-\int_{M} \mathrm{~A} \cdot \nabla \mathbf{w} d \mu_{1} .
$$

Since $\mathbf{w}\left(x_{0}\right)=0$ and $\nabla \mathbf{w}\left(x_{0}\right)=0$, taking into account Theorem 3 and (c) of Definition 3 one has

$$
\lim _{i \rightarrow \infty} \frac{\lambda\left(M_{i}\right)}{\eta\left(M_{i}\right)}=0,
$$

for $\eta$-a.e. $x_{0} \in \Omega$, hence $\lambda=0$.

From now on, we shall denote with the same symbol $P$ such an extension. 


\section{Virtual stress power}

We recall that a vector field $\mathbf{v}$ is rigid if $\nabla \mathbf{v}$ is skew. By a virtual stress power (or virtual power of internal forces) we mean a power $P_{(i)}$ of order one which satisfies the following axiom:

Axiom 1 (Axiom of virtual stress powers) $P_{(i)}(M, \mathbf{v})$ is null for every rigid velocity field $\mathbf{v} \in$ Aff and every $M \in \mathscr{M}_{G}$.

This is equivalent to state that $P_{(i)}$ is an objective quantity, i.e. it has the same value in every reference frame.

In view of the previous axiom, the stress power admits a special representation. First of all, since $P_{(i)}$ is a power of order one, by Theorem 4 one has

$$
P_{(i)}(M, \mathbf{v})=\int_{M} \mathbf{a} \cdot \mathbf{v} d \mu_{0}+\int_{M} \mathrm{~A} \cdot \nabla \mathbf{v} d \mu_{1}
$$

Let us denote with $\mathrm{D}$ the symmetric part of $\nabla \mathbf{v}$, i.e. $\mathrm{D}=(\nabla \mathbf{v})^{s}$.

Theorem 6 Let $P_{(i)}$ be a virtual stress power. Then

$$
P_{(i)}(M, \mathbf{v})=-\int_{M} \mathrm{~T} \cdot \mathrm{D} d \mu_{1}
$$

for every $M \in \mathscr{M}_{G}$ and $\mathbf{v} \in \mathrm{Aff}$, where $\mathrm{T}=-\mathrm{A}^{s}$ is symmetric.

Proof Consider a constant vector field $\mathbf{v}_{0} \in \mathbb{R}^{n}$. Since

$$
\forall M \in \mathscr{M}_{G}: \quad P_{(i)}\left(M, \mathbf{v}_{0}\right)=\int_{M} \mathbf{a} \cdot \mathbf{v}_{0} d \mu_{0}
$$

and the left-hand side vanishes in view of Axiom 1, the arbitrariness of $\mathbf{v}_{0}$ implies

$$
\forall M \in \mathscr{M}_{G}: \quad \int_{M} \mathbf{a} d \mu_{0}=0
$$

Hence by Corollary 1 one has $\mathbf{a}=0 \mu_{0}$-a.e. and

$$
\forall M \in \mathscr{M}_{G}: \quad P_{(i)}(M, \mathbf{v})=\int_{M} \mathrm{~A} \cdot \nabla \mathbf{v} d \mu_{1} .
$$

Now consider a skew matrix $F \in \operatorname{Lin}$ and the vector field $\mathbf{v}(x)=\mathrm{F} x$. From (7) and Axiom 1 it follows that

$$
\forall M \in \mathscr{M}_{G}: \quad \int_{M} \mathrm{~A} \cdot \mathrm{F} d \mu_{1}=\int_{M} \mathrm{~A}^{\omega} \cdot \mathrm{F} d \mu_{1}=0
$$

and the arbitrariness of $F$ yields $A^{\omega}=0 \mu_{1}$-a.e. 


\section{Virtual contact powers}

In this brief section we investigate some features of the contact powers. In the standard doctrine of Continuum Mechanics, the contact power is defined as the resultant of the stress vector field over the boundary of a subbody. In the present, more general, situation where the existence of a stress field is not assumed a priori, we assume that the contact power is a particular power of order one satisfiyng an additional assumption of distributional flavor. The simple idea is that one wants a contact power to act only on the boundary of a subbody.

Let $P$ be a power of order one with

$$
P(M, \mathbf{v})=\int_{M} \mathbf{b} \cdot \mathbf{v} d \gamma_{0}+\int_{M} \mathrm{~B} \cdot \nabla \mathbf{v} d \gamma_{1}
$$

Definition 4 We say that $P$ is a contact power, if

$$
\forall M \in \mathscr{M}_{G}, \forall \mathbf{v} \in C_{0}^{\infty}\left(\Omega ; \mathbb{R}^{n}\right): \quad \operatorname{supt} \mathbf{v} \subseteq M \Rightarrow P(M, \mathbf{v})=0 .
$$

Note that even if a power of order one is defined on affine virtual velocities, the previous assumption makes sense in view of the Extension Theorem 5.

The following is a distributional characterization of contact powers.

Theorem 7 Let $P$ be a power of order one. Then $P$ is a contact power if and only if

$$
\mathbf{b} \gamma_{0}=\operatorname{div}\left(B \gamma_{1}\right)
$$

on $\Omega$ in the sense of distributions.

Proof Consider $\mathbf{v} \in C_{0}^{\infty}\left(\Omega ; \mathbb{R}^{n}\right)$ and let $M \in \mathscr{M}_{G}$ be such that supt $\mathbf{v} \subseteq M$. One has

$$
\left\langle\mathbf{b} \gamma_{0}, \mathbf{v}\right\rangle=\int_{\Omega} \mathbf{b} \cdot \mathbf{v} d \gamma_{0}=\int_{M} \mathbf{b} \cdot \mathbf{v} d \gamma_{0}
$$

and, by definition of distributional divergence,

$$
\left\langle\operatorname{div}\left(\mathrm{B} \gamma_{1}\right), \mathbf{v}\right\rangle=-\int_{\Omega} \mathrm{B} \cdot \nabla \mathbf{v} d \gamma_{1}=-\int_{M} \mathrm{~B} \cdot \nabla \mathbf{v} d \gamma_{1}
$$

Then it is clear that $P$ is a contact power if and only if the left-hand sides do coincide.

\section{The principle of virtual powers}

From now on we will assume that

$$
P_{(d)}(M, \mathbf{v})=\int_{M} \mathbf{a} \cdot \mathbf{v} d \mu_{0}+\int_{M} \mathrm{~A} \cdot \nabla \mathbf{v} d \mu_{1}
$$


is a given power of order one, which will be called power of external forces. Moreover $P_{(c)}$ will be the contact power and $P_{(i)}$ the stress power. In particular, by Theorems 4 and 6 they can be represented as

$$
\begin{gathered}
P_{(c)}(M, \mathbf{v})=\int_{M} \mathbf{b} \cdot \mathbf{v} d \gamma_{0}+\int_{M} \mathrm{~B} \cdot \nabla \mathbf{v} d \gamma_{1}, \\
P_{(i)}(M, \mathbf{v})=-\int_{M} \mathrm{~T} \cdot \mathrm{D} d v,
\end{gathered}
$$

where $\mathrm{T}$ is symmetric.

The following axiom is the classical formulation of the principle of virtual powers in the case of Continuum Mechanics.

Axiom 2 (Principle of virtual powers) Denoting with $P_{(d)}$ the power of external forces, with $P_{(i)}$ the stress power and with $P_{(c)}$ the contact power, we assume that a necessary and sufficient condition for a continuous body to be in equilibrium is

$$
P_{(d)}(M, \mathbf{v})+P_{(i)}(M, \mathbf{v})+P_{(c)}(M, \mathbf{v})=0
$$

for every $M \in \mathscr{M}_{G}$ and every virtual velocity $\mathbf{v} \in$ Aff.

Remark 2 Although the previous axiom is an equilibrium condition, by a standard implementation of the so called d'Alembert principle it can cover also the dynamical case: it is sufficient to keep into account also of the inertial terms into the power of external forces.

Remark 3 Due to the nature of our presentation, which stops at first order powers, it seems natural to assume the previous principle for affine virtual velocity fields. However, it can be proved that it is equivalent to assume Axiom 2 on the class of $C^{1}$ virtual velocities. Indeed, as we have seen in Theorem 5 a power of order one is uniquely determined by its behavior on affine vector fields.

Then the Principle of virtual powers becomes

$$
\int_{M} \mathbf{a} \cdot \mathbf{v} d \mu_{0}+\int_{M} \mathrm{~A} \cdot \nabla \mathbf{v} d \mu_{1}-\int_{M} \mathrm{~T} \cdot \mathrm{D} d v+\int_{M} \mathbf{b} \cdot \mathbf{v} d \gamma_{0}+\int_{M} \mathrm{~B} \cdot \nabla \mathbf{v} d \gamma_{1}=0
$$

for every $M \in \mathscr{M}_{G}$ and $\mathbf{v} \in$ Aff.

Consider a constant field $\mathbf{v}(x)=\mathbf{v}_{0}$; one has

$$
\forall \mathbf{v}_{0} \in \mathbb{R}^{n}, \forall M \in \mathscr{M}_{G}: \quad \int_{M} \mathbf{a} \cdot \mathbf{v}_{0} d \mu_{0}+\int_{M} \mathbf{b} \cdot \mathbf{v}_{0} d \gamma_{0}=0
$$

and Corollary 1 yields the identity of measures

$$
\mathbf{a} \mu_{0}+\mathbf{b} \gamma_{0}=0 .
$$

In particular, by Theorem 7 one obtains

$$
\mathbf{a} \mu_{0}+\operatorname{div}\left(\mathrm{B} \gamma_{1}\right)=0 .
$$

Moreover (8) becomes

$$
\int_{M} \mathrm{~A} \cdot \nabla \mathbf{v} d \mu_{1}-\int_{M} \mathrm{~T} \cdot \mathrm{D} d v+\int_{M} \mathrm{~B} \cdot \nabla \mathbf{v} d \gamma_{1}=0
$$


for every $M \in \mathscr{M}_{G}$ and $\mathbf{v} \in$ Aff. Taking the symmetric and skew-symmetric part of the tensor fields, keeping into account the arbitrariness of $\mathbf{v}$ and $M$ one gets

$$
\mathrm{B} \gamma_{1}=\mathrm{T} v-\mathrm{A} \mu_{1},
$$

where, in particular,

$$
\mathrm{B}^{s} \gamma_{1}=\mathrm{T} v-\mathrm{A}^{s} \mu_{1}, \quad \mathrm{~B}^{\omega} \gamma_{1}=-\mathrm{A}^{\omega} \mu_{1} .
$$

Finally, by combining (9) and (10) one obtains

$$
\mathbf{a} \mu_{0}+\operatorname{div}\left(\mathrm{T} v-\mathrm{A} \mu_{1}\right)=0 .
$$

The following situations are remarkable:

- If the power of external forces has order 0 (i.e. $A=0$ ) then $B$ is symmetric $\gamma_{1}$-a.e., $\mathrm{T} v+\mathrm{B} \gamma_{1}=0$ and the standard balance equation

$$
\mathbf{a} \mu_{0}+\operatorname{div}(T v)=0
$$

is achieved.

- B is symmetric $\gamma_{1}$-a.e. if and only if A is symmetric $\mu_{1}$-a.e.

\section{Boundary powers}

We study now a special case, where the contact power is a set function bounded by an integral on the boundary of the subbody. In the spirit of the paper, the involved measure is not assumed to be the area measure. A similar approach were adopted in [6].

Definition 5 A boundary power is a function

$$
P_{(c)}: \mathscr{M}_{G} \times C_{0}^{\infty}\left(\Omega ; \mathbb{R}^{n}\right) \rightarrow \mathbb{R},
$$

such that the following properties hold:

1. for every $\mathbf{v} \in C_{0}^{\infty}\left(\Omega ; \mathbb{R}^{n}\right), P_{(c)}(\cdot, \mathbf{v})$ is $*$-additive;

2. for every $M \in \mathscr{M}_{G}, P_{(c)}(M, \cdot)$ is linear;

3. for every $M \in \mathscr{M}_{G}$ there exists $\gamma_{M} \in \mathfrak{M}(\Omega)$ such that

$$
\forall \mathbf{v} \in C_{0}^{\infty}\left(\Omega ; \mathbb{R}^{n}\right): \quad\left|P_{(c)}(M, \mathbf{v})\right| \leqslant \int_{\mathrm{bd} M}|\mathbf{v}| d \gamma_{M} .
$$

Proposition 2 Let $P$ be a boundary power. Then for every $M \in \mathscr{M}_{G}$ there exists a Borel vector field $\mathbf{t}_{M}: \Omega \rightarrow \mathbb{R}^{n}$ such that

$$
P(M, \mathbf{v})=\int_{\mathrm{bd} M} \mathbf{t}_{M} \cdot \mathbf{v} d \gamma_{M}
$$

for every $\mathbf{v} \in C_{0}^{\infty}\left(\Omega ; \mathbb{R}^{n}\right)$. 
Proof Let $M \in \mathscr{M}_{G}$. The map $P(M, \cdot): C_{0}^{\infty}\left(\Omega ; \mathbb{R}^{n}\right) \rightarrow \mathbb{R}$ is linear and

$$
|P(M, \mathbf{v})| \leqslant\|\mathbf{v}\|_{\infty} \gamma_{M}(\operatorname{bd} M),
$$

hence it is a vector distribution on $\Omega$ of order zero. Moreover it is absolutely continuous with respect to $\gamma_{M}\llcorner\mathrm{bd} M$ and the proof is complete.

In the classical situation the measure $\gamma_{M}$ is simply the area measure $\mathscr{H}^{n-1}$ restricted to bd $M$ and $\mathbf{t}_{M}$ is the stress vector field, which depends on $M$ only through the surface normal.

If the principle of virtual powers 2 is assumed, one has

$$
\left|P_{(c)}(M, \mathbf{v})\right|=\left|P_{(d)}(M, \mathbf{v})+P_{(i)}(M, \mathbf{v})\right| \leqslant \int_{M}|\mathbf{v}| d \mu_{0}+\int_{M}|\nabla \mathbf{v}| d\left(\mu_{1}+v\right) .
$$

Hence in particular a boundary power is a power of order one. Moreover, assumption (c) implies that $P_{(c)}(M, \mathbf{v})=0$ whenever supt $\mathbf{v} \subseteq M$, hence a boundary power is a contact power and we can apply the results of section 6 .

A very interesting result can be obtained if we slightly increase the regularity for $P_{(i)}$ and $P_{(d)}$. Namely, we assume that $\mu_{1}=v=\mathscr{L}^{n}$ and that A, T be tensor fields with divergence measure. In this case there exists a full grid, denoted again with $G$, such that

$$
\begin{aligned}
\int_{M} \mathrm{~A} \cdot \nabla \mathbf{v} d \mathscr{L}^{n} & =\int_{\mathrm{bd} M} \mathrm{~A} \mathbf{n}^{M} \cdot \mathbf{v} d \mathscr{H}^{n-1}-\int_{M} \mathbf{v} \cdot \operatorname{div} \mathrm{A} \\
\int_{M} \mathrm{~T} \cdot \mathrm{D} d \mathscr{L}^{n} & =\int_{\mathrm{bd} M} \mathrm{Tn}^{M} \cdot \mathbf{v} d \mathscr{H}^{n-1}-\int_{M} \mathbf{v} \cdot \operatorname{div} \mathrm{T}
\end{aligned}
$$

for every $M \in \mathscr{M}_{G}$ and every $\mathbf{v} \in C^{1}\left(\Omega ; \mathbb{R}^{n}\right)$. Moreover, equation (11) becomes

$$
a \mu_{0}+\operatorname{div} \mathrm{T}-\operatorname{div} \mathrm{A}=0 .
$$

By stating the Principle of virtual powers we obtain the integral equation

$$
\int_{\mathrm{bd} M} \mathrm{~A} \mathbf{n}^{M} \cdot \mathbf{v} d \mathscr{H}^{n-1}-\int_{\mathrm{bd} M} \mathrm{Tn}^{M} \cdot \mathbf{v} d \mathscr{H}^{n-1}+\int_{\mathrm{bd} M} \mathbf{t}_{M} \cdot \mathbf{v} d \gamma_{M}=0,
$$

which holds for every $M \in \mathscr{M}_{G}$ and $\mathbf{v} \in$ Aff. Now the arbitrariness of $\mathbf{v}$ yields $\gamma_{M} \ll \mathscr{H}^{n-1}\llcorner\mathrm{bd} M$ and

$$
\mathbf{t}_{M}=(\mathrm{T}-\mathrm{A}) \mathbf{n}^{M},
$$

which is a general version of the Cauchy Stress Theorem.

\section{References}

1. Degiovanni M., Marzocchi A., Musesti A.: Edge-force densities and second-order powers. Ann. Matem. Pura Appl. 185 (1), 81-103 (2006)

2. Evans L. C., Gariepy R. F.: Measure theory and fine properties of functions. CRC Press, Boca Raton (1992)

3. Federer H.: Geometric Measure Theory. Springer-Verlag, New York (1969)

4. Germain P.: La méthode des puissances virtuelles en mécanique des milieux continus. Première partie. Théorie du second gradient. J. Mécanique 12, 235-274 (1973)

5. Germain P.: The method of virtual power in continuum mechanics. Part 2: Microstructure. SIAM J. Appl. Math. 25, 556-575 (1973)

6. Marzocchi A., Musesti A.: Balanced virtual powers in Continuum Mechanics. Meccanica 38, 369-389 (2003)

7. Ruggeri T.: Sulla meccanica delle microstrutture. Istit. Veneto Sci. Lett. Arti Atti Cl. Sci. Mat. Natur. 129, 257-271 (1971) 